\title{
Obstáculos para la igualdad de género en las universidades
}

\author{
Obstacles to gender equality in universities
}

\author{
Capitolina Díaz Martínez \\ Universidad de Valencia \\ capitolina.diaz@uv.es
}

\section{Resumen}

Las mujeres, llevando sólo un siglo en las universidades, han alcanzado cotas de rendimiento equivalentes a las masculinas. Sin embargo, en nuestras universidades permanece y prevalece una cultura androcéntrica y los equipos rectores no lo consideran un problema. La identificación de los principales rasgos estructurales que obstaculizan y frenan el desarrollo en igualdad de las universitarias y los universitarios junto a algunas propuestas de intervenciones para mejorar esta discriminación, centran este artículo. Entre los rasgos negativos analizados y para los que se sugieren propuestas de cambio están: el acoso sexual y la violencia de género, la falta de medidas para la conciliación de la vida universitaria y la vida personal y el androcentrismo en los contenidos docentes y de investigación. Se plantea, así mismo, la necesidad de presupuestos con perspectiva de género y algunas consecuencias derivadas de los obstáculos señalados, como la brecha salarial de género en las universidades y el techo de cristal.

Palabras clave: defensoría universitaria, sesgos de género, discriminación de género estructural, cultura androcéntrica, histéresis de género.

\begin{abstract}
Women, having only been in college for a century, have reached performance levels equivalent to men's. However, an androcentric culture remains and prevails in our universities and the governing teams do not consider it a problem. The identification of the main structural features that hinder and slow down the development of equality among university women and men, together with some proposals for interventions to improve this discrimination, focus this article. Among the negative traits that we analyse and for which we offer proposals for change are: sexual harassment and gender violence, the lack of measures to reconcile university life and personal life, and androcentrism in teaching and research content. Likewise, we argue in favour of budgets with a gender perspective discuss some consequences derived from the mentioned obstacles, such as the gender pay gap in universities and the glass ceiling.
\end{abstract}

Keywords: university ombudsman, gender biases, structural gender discrimination, androcentric culture, gender hysteresis.

Sumario. Introducción. 1-Los sesgos implícitos. 2-El acoso (sexual y por razón de sexo o género) y la violencia de género. 3-Las difcultades para la conciliación de la vida laboral y personal-familiar. 4-Los contenidos androcéntricos de la ciencia y de la docencia. 5-La brecha salarial de género. 6-La escasa cultura de igualdad. 7-Referencias. 


\section{Introducción}

Las defensorías universitarias están teniendo un papel muy importante en la cultura académica española. Las defensorías son el mecanismo administrativo que indica al personal universitario que no está sólo. Son aquello que la universidad ha creado para protegerse de sí misma. Para protegerse de los errores que alguien en la universidad pueda cometer y para ampliar los límites que sus estructuras presentes imponen al personal universitario. Las estructuras universitarias, como cualquier otra estructura, empiezan a envejecer nada más crearse. Por eso, lo más sabio es dotar a las estructuras de mecanismos de vigilancia y actualización de sí mismas. En ese sentido, la creación de las defensorías ha sido una acción inteligente y proactiva.

Es precisamente en el nivel estructural donde la Defensoría tiene más tareas pendientes en el ámbito tratado en este artículo. A saber, las desigualdades de sexo y género. Las universidades, como otras instituciones, se han creado sobre bases androcéntricas o masculino-céntricas, para entendernos mejor. Como bien sabemos, en su origen nada más albergaban hombres y han tardado mucho en aceptar mujeres, y con reticencias, como indica María Ángeles Durán en su pionero trabajo Una ausencia de mil años: la mujer en la universidad. Así, hasta 1910 las universidades españolas no permitieron entrar, con pleno derecho, a las mujeres en sus aulas. Y en algunos colleges de Oxford no las han aceptado hasta $1974^{1}$. Con esta presencia de más de un siglo las mujeres han mostrado un rendimiento a la altura del de los hombres (en cualquier percentil). Pero los sesgos de género, los estereotipos sexistas y la obsolescencia estructural (lo que en otro lugar hemos llamado "histéresis de género" (Díaz y Dema, 2013) perviven. Estas muestras de sexismo hacen que nuestras universidades todavía muestren numerosas brechas de género (por cierto, no más que otras instituciones y menos que algunas de ellas). Diversas investigaciones constatan brechas salariales (Jabbaz, Samper y Díaz, 2019; Jubeto y Larrañaga, 2017); brechas de poder como el conocido efecto "techo de cristal" (Parlamento Europeo, 2014) y la disparidad de género en las cátedras ${ }^{2}$; androcentrismo en los contenidos docentes e investigadores (Nuño Gómez y Álvarez Conde, 2017); falta de medidas que compensen la brecha de cuidados que lastra el tiempo de trabajo de las académicas y amplía el de los académicos (Selma, 2019), etc. etc.

Estas estructuras universitarias androcéntricas, si no se revisan desde el punto de vista y las experiencias de las mujeres y de otras personas con entidades de género diversas, se seguirán tomando como naturales y neutrales en relación con el sexo y el género. Pero esa revisión está por hacer y la institución universitaria muestra notables sesgos de género. En efecto, sólo el sexismo y la discriminación de género-sexo, casi siempre sutil y encubierta, pueden explicar las diferencias de logro entre mujeres y hombres en la carrera académica. Dichas diferencias tanto de las universidades españolas (gráfico 1) como de las europeas (EU 28) quedan bien reflejadas en el diagrama de tijera de este gráfico.

\footnotetext{
${ }^{1}$ http://www.ox.ac.uk/about/oxford-people/women-at-oxford

2 Los frenos a las mujeres para alcanzar las cátedras son tan evidentes que el Tribunal Supremo (Sala III de lo Contencioso-Administrativo) ha fallado a favor de que las universidades incluyan una variable de género para convocar nuevas cátedras. Los hombres podrán competir por ellas en igualdad de condiciones.

http://www.poderjudicial.es/cgpj/es/Poder-Judicial/Tribunal-Supremo/Noticias-Judiciales/El-Tribunal-Supremo-avala-que-las-universidades-incluyan-una-variable-de-genero-en-los-criterios-para-seleccionarlos-departamentos-donde-crear-nuevas-catedras
} 
Gráfico 1. Proporción (\%) de hombres y mujeres en una carrera académica típica, estudiantes y PDI,

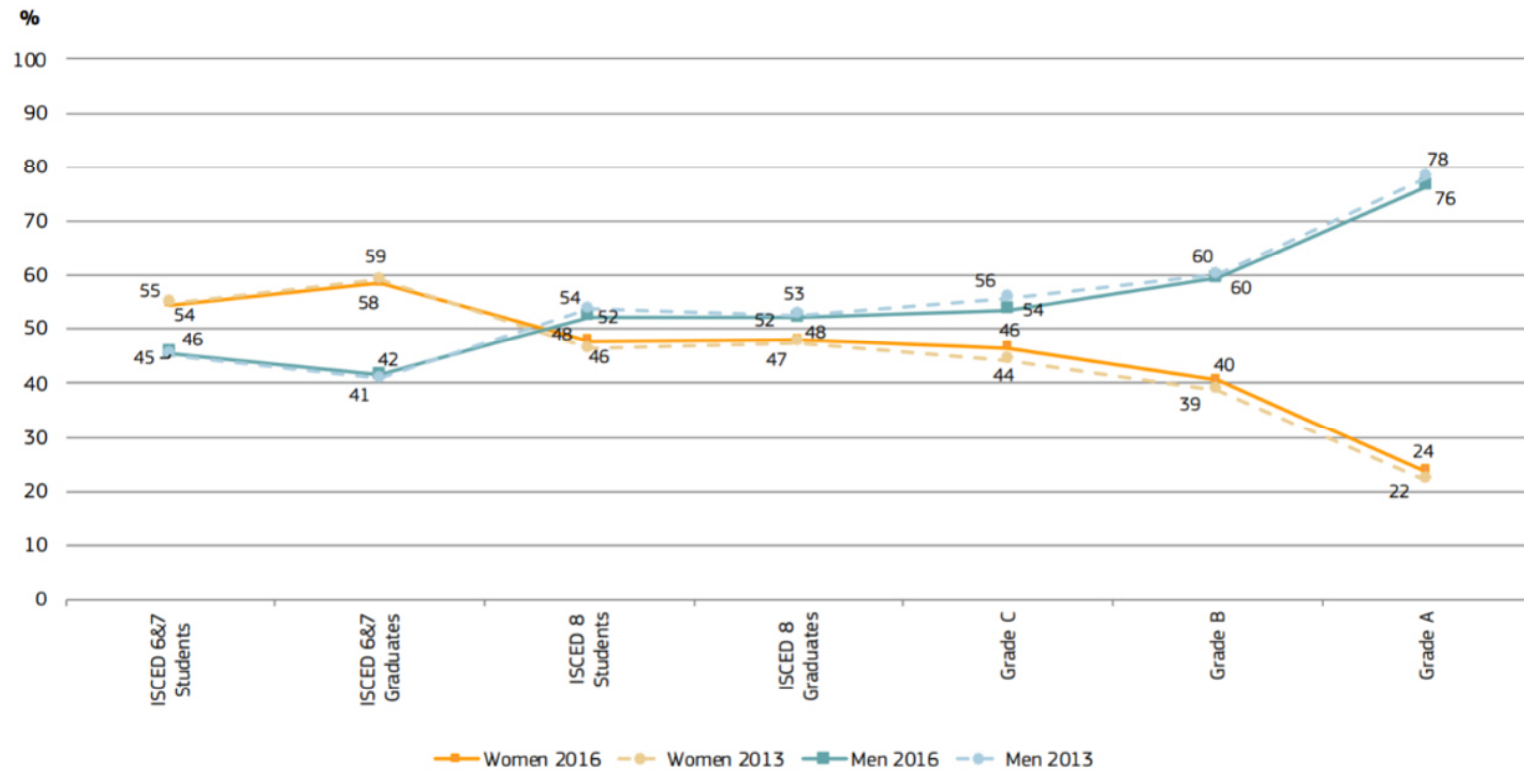

Fuente: Shefigures, 2016

Podemos resumir estas diferencias con los datos siguientes: en España (según Científicas en Cifras, 2017, gráfico 2), el 58\% de quienes finalizan sus estudios de grado son mujeres, pero obtienen el $49 \%$ de los doctorados; son el $48 \%$ de TU y sólo llegan al $21 \%$ de las cátedras. En la EU, según Shefigures, 2018, hay un $21 \%$ catedráticas y un $18 \%$ de rectoras o equivalente (gráfico 1).

Gráfico 2. Distribución de mujeres y hombres en la carrera investigadora en centros propios de las universidades públicas. Curso 2016/17

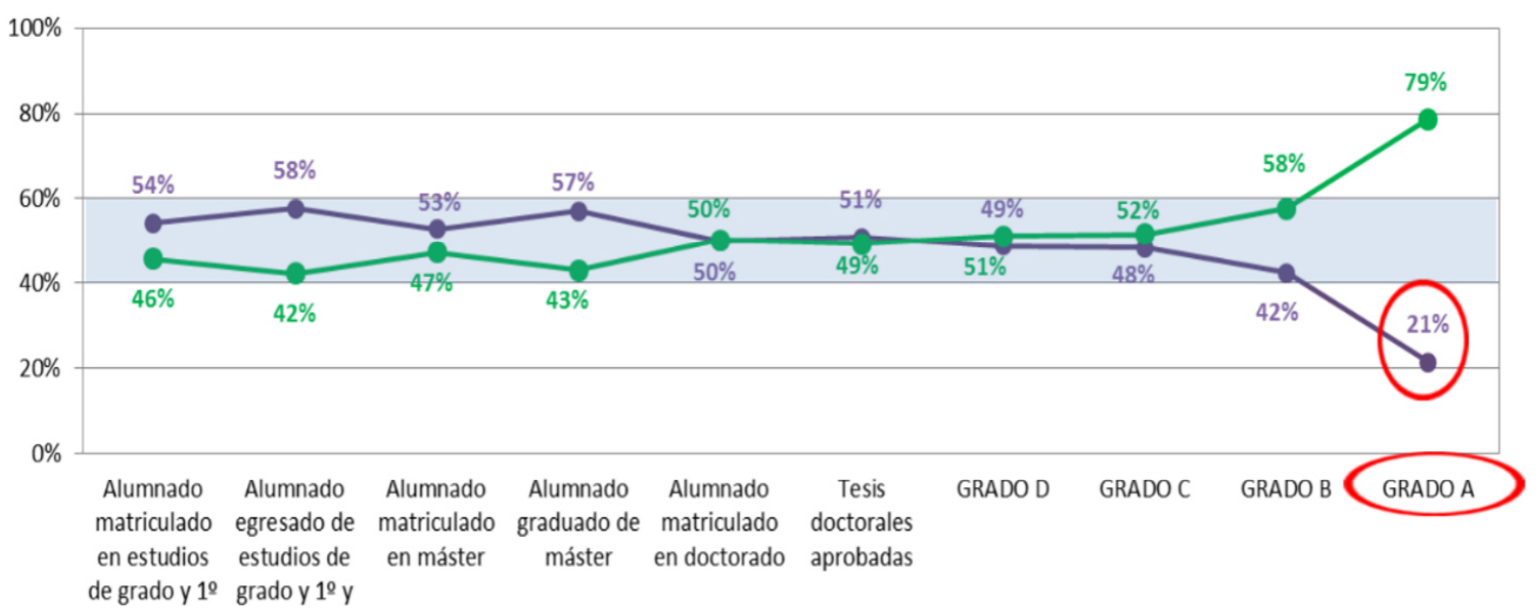

Porcentaje de mujeres y hombres sobre el total de cada categoría. Fuente: Científicas en Cifras, 2017.

¿Qué factores pueden explicar esta pérdida de talento femenino? ¿Por qué entran tantas jóvenes mujeres en la universidad, rinden bien y luego se pierden para la academia? Nos centraremos en seis grandes causas sobre las que, a nuestro entender, las Defensorías podrían intervenir, tanto a demanda como de oficio. 


\section{Los sesgos implícitos}

Nos dice la Real Academia de la Lengua que sesgar es torcer algo a un lado. Si torcemos el conocimiento habrá lados que no veamos y aquellos que podemos ver estarán marcados por lo oblicuo de la torcedura. Nuestro pensamiento, de forma casi indetectable, pero con una incansable persistencia, está dominado por numerosos sesgos implícitos. Todas las personas tenemos sesgos. Hombres contra mujeres y viceversa; personas cisgénero contra personas transgénero y viceversa; por razones de etnia, credo político o religioso, forma corporal, etc. (Marks, M. J., \& Fraley, R. C. 2006; Owad, T., 2006; The World Bank, 2015). Tenemos sesgos, forman parte de la manera de aprehender el mundo. Lo importante es saberlo para mantenerse alerta y poner remedio a sus efectos perniciosos. Especialmente en ámbitos como el de la ciencia en el cual, el sesgar el conocimiento, tomado como neutral y científico, nos está sesgando las bases mismas de lo que sabemos de nosotros como seres humanos y del mundo que nos rodea. Algunas instituciones ya empiezan a darse cuenta de ello. La publicación en 2018 Lo que sí funciona de Iris Bohnet ${ }^{3}$, responsable de Women and Public Policy Program (WPPP) de la Kennedy School for Government de la Universidad de Harvard ha sido un aldabonazo para esa propia universidad y debiera serlo para otras que desearan emular los estándares de excelencia académica de esa universidad. Por su parte, y algo más cerca, la Liga Europea de Universidades de Investigación (LERU) en su Position Paper, Sept, 2019 , indica que la primera medida a tomar, en esta dirección, es que el equipo rector reconozca la existencia ubicua de sesgos de género y se decida públicamente a ponerles freno ${ }^{4}$. ¿Cómo proponen resolverlo? Con un programa de entrenamiento anti-sesgo que asegure la presencia de personas expertas en género en los lugares de toma de decisiones y mediante la revisión de los presupuestos desde la perspectiva de género,

Gráfico 3. Distribución de mujeres y hombres en órganos unipersonales de gobierno de las universidades públicas, 2017.

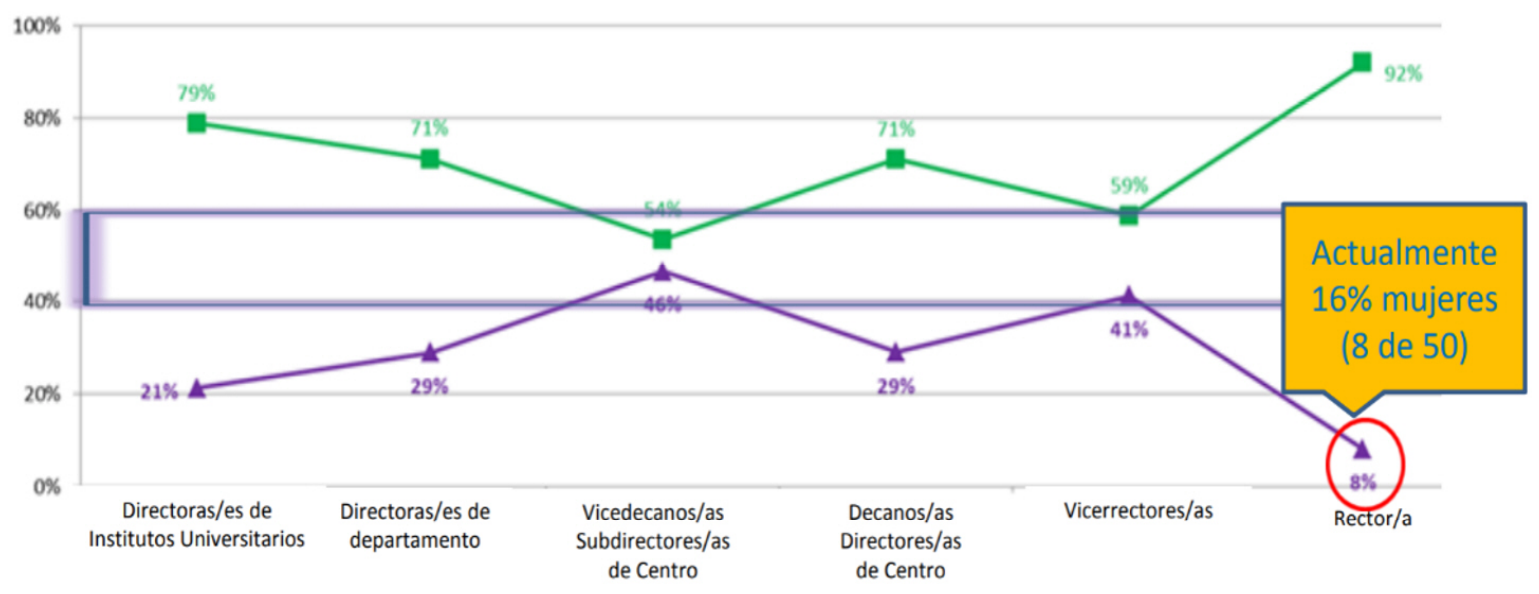

Porcentaje de mujeres y hombres sobre el total de cada categoría. Fuente: Científicas en Cifras, 2017.

\footnotetext{
3 Un breve y concentrado resumen del libro se puede ver en https://www.youtube.com/watch?time_continue= $16 \& \mathrm{v}=$ niH9wfKsUIc\&feature $=$ emb_title

4 www.euroscientist.com/implicit-bias-can-undermine-academic- meritocracy/?utm_source=EuroScientist\&utm_campaign=984bb09c4d-EMAIL_CAMPAIGN_2018_01_10\&utm_medium=email\&utm_term=0_ 8c57a1a873-984bb09c4d-125977481
} 
etc. Estos sesgos de género insertos en las estructuras universitarias se suman, en una sinergia negativa, a los propios sesgos del entorno social, tales como la dificultad de conciliar la vida profesional y la personal. Unidos explican el techo de cristal con el que se encuentran las académicas (gráfico3) y el suelo pegajoso que les dificulta elevarse a las alturas conseguidas por sus colegas masculinos.

Una vez subrayada la capilaridad de los sesgos y la necesidad de la autoconciencia institucional sobre ellos, veamos los principales problemas sexistas que detectamos en nuestras universidades (en las nuestras, las españolas, y en mayor o menor medida, también las de fuera de nuestras fronteras).

2. El acoso (sexual y por razón de sexo o género) y la violencia de género

The unnamed should not be mistaken for the non-existent. MacKinnon 1979, 27-28.

El acoso sexual (en el sentido amplio de avances no deseados con connotaciones sexuales) es un problema en cierta medida oculto pero omnipresente, y tiene un coste devastador para el bienestar y el quehacer de muchas personas en nuestras sociedades. Las universidades no son ajenas a este desgraciado fenómeno y hay suficiente literatura científica que lo confirma (Benson y Thomson, 1982; Reilly, Lott, B. \& Gallogly, 1986; Dessel, A. B., K. D. Goodman, and M. R. Woodford. 2017; Johnson, P., S. Widnall, and B. Frazier, 2018; Unidad de Igualdad UCM, 2018; y SWG GRI. 2020, entre otros). Pero se ha investigado mucho menos sobre programas específicos de capacitación que pueden ayudar a reducir el acoso sexual y la violencia de género ${ }^{5}$. Esos programas debieran diseñarse especialmente para ayudar a las víctimas, pero convendría que incorporaran, también, propuestas para tratar a los culpables y a los potencialmente perpetradores.

Respecto a su carácter generalizado y las causas que lo motivan, Bondestam y Lundqvist (2020a) del Secretariado Sueco para la Investigación de Género, señalan: El acoso sexual es una epidemia en todo el mundo, impacta a los sistemas educativos e impacta a los individuos, grupos y cualquier clase de organización de manera profunda. Condiciones de trabajo precarias, organizaciones jerárquicas, normalización de la violencia de género, masculinidades académicas tóxicas, una cultura del silencio y la falta de liderazgo activo son características clave que permiten el acoso sexual.

Ante las quejas de acoso sexual y violencia de género, nuestras universidades todavía muestran una tendencia hacia una primera reacción negacionista, seguida de una de minusvaloración. Ello se debe a que aún no han tomado conciencia del problema, de cómo este afecta a su personal y de que hay formas de ponerle remedio. Pero es justo reconocer que han alcanzado un cierto nivel de reconocimiento del problema. Este reconocimiento ha permitido que, prácticamente todas ellas, tengan protocolos de actuación en caso de acoso sexual y por razón de sexo o género y contra la violencia de género y la homofobia. Así todo, no ha sido sencillo aprobar esos protocolos. El esfuerzo que han realizado las impulsoras de tales protocolos merecería un tratado en sí mismo. Los protocolos, resul-

\footnotetext{
5 Algunas propuestas pueden encontrarse en https://www.qs.com/how-universities-provide-best-supportservices-victims-sexual-harassment/ ; https://www.apa.org/monitor/2018/05/sexual-harassment o en https://www.universitiesaustralia.edu.au/wp-content/uploads/2019/05/UA-Guidelines-2.pdf; SWG GRI. 2020 y Bondestam and Maja Lundqvist (2020b).
} 
tado de ese esfuerzo, evidencian las limitaciones derivadas de las estructuras y prácticas universitarias androcéntricas que son bastante visibles en buena parte de ellos ${ }^{6}$. Un buen número de los protocolos es excesivamente garantista para el personal de la universidad. Además, en la medida en que los protocolos no tienen capacidad disciplinaria, una vez realizadas las actuaciones y en caso de responsabilidad verificada, hay que acudir al régimen disciplinario funcionarial, más garantista si cabe, lo que alarga el proceso, puede revictimizar a las denunciantes y, según algunas interpretaciones, deja sin voz a las víctimas por no considerarlas parte del proceso. La situación se empeora porque, con frecuencia, las personas encargadas de las instrucciones de los casos de denuncia por acoso, violencia o LGTBi-fobia carecen de formación en género y en violencia machista. La falta de liderazgo activo, a este respecto, por parte de las autoridades universitarias, no facilita la difusión de los protocolos cuya existencia no es suficientemente conocida ni por el alumnado ni por el personal (PDI y PAS).

Las defensorías, en combinación con las unidades de igualdad, podrían ser un instrumento de divulgación de la existencia de los protocolos contra el acoso y la violencia. Si así lo entendieran, podrían no sólo favorecer que se conocieran los protocolos sino, también, promover la existencia de "puntos violeta" o equivalentes en los cuales sea fácil comunicar los casos de acoso y violencia sin dificultades y de manera informal para las personas involucradas. Esos puntos sirven para que, sin reducir el rigor que esos asuntos merecen, las víctimas puedan comunicar sus problemas de manera amistosa y discreta (si así se desea). Dichos puntos son precisos porque, es sabido, que el acoso y la violencia de género inhiben a las víctimas de la búsqueda de ayuda. Un complemento de esta medida podría ser la facilitación de redes de apoyo mutuo entre víctimas.

En esta misma línea, las defensorías podrían, junto con las Unidades de Igualdad (o equivalentes) que estén dispuestas a ello, auspiciar la creación de espacios de trabajo con alumnado y personal universitario masculino. Este tipo de iniciativas están muy poco extendidas e incluso hay notables resistencias a crearlas. Pero, como sostiene Brendan L. Smith en un artículo publicado en el boletín de la Asociación Americana de Psicología (APA) en 2018: No se trata solo del problema de una mujer. Las mujeres continúan siendo las principales víctimas del acoso sexual y llevan la carga del sufrimiento. [... pero] hasta que los hombres no asuman su responsabilidad en el problema, será realmente difícil lograr un gran movimiento para abordarlo.

Hemos de asumir que los hombres han nacido, se han socializado y han sido educados en una sociedad de supremacía masculina. Una sociedad donde los modelos de masculinidad son poco igualitarios (por decirlo suave). Hay hombres que no han sabido o no han podido desconstruir el modelo recibido y reconstruir una masculinidad igualitaria y respetuosa con otros géneros. A estos hombres, sería conveniente ofrecerles oportunidades para entenderse a sí mismos y entender, a la vez, las relaciones de género(s) de forma no discriminatoria, no tóxica y no violenta. De no brindarles oportunidades para el cambio, es muy difícil que espontáneamente lo hagan y, consecuentemente, reiterarán sus pautas de comportamiento machistas. En resumen, parece conveniente ofertar en nuestras uni-

\footnotetext{
6 Hasta donde yo conozco no hay publicaciones que recojan las enormes dificultades que las directoras de Unidades de Igualdad han sufrido tratando que sus universidades aprobaran los Protocolos contra el acoso sexual y por razón de género y sexo. Pero el desempeño de mi cargo, primero como directora de la Unidad de Mujeres y Ciencia (Ministerio de Educación y Ciencia) y después como presidenta de la Asociación de Mujeres Investigadoras y Tecnólogas (AMIT) me ha permitido constatar lo que digo.
} 
versidades, a los hombres, y en especial a los hombres jóvenes, espacios de acogida en los cuales puedan mostrar sus dudas, dificultades y temores. Espacios y oportunidades en los que, desde el reconocimiento de lo que puede considerarse como un déficit actitudinal por su parte, puedan abrirse a fórmulas de desarrollo conductual basado en el respeto mutuo y en el aprendizaje de relaciones de género constructivas.

La Organización Mundial de la Salud (Barker, Gary, Christine Ricardo y Marcos Nascimento 2007), lleva años haciendo propuestas de incorporar a los hombres y los niños en la defensa de la igualdad; nuestras universidades también podrían empezar a hacerlo.

No me cabe duda de que lo principal es que nuestras universidades sean lugares seguros, libres de acoso y violencia. Si me he extendido en la importancia de trabajar con perpetradores (reales o potenciales) obedece a la desconsideración que merecen por parte de la mayoría de las instituciones.

\section{Las dificultades para la conciliación de la vida laboral y personal-familiar}

El trabajo docente e investigador, como buena parte de los trabajos de alta cualificación en el mundo entero, se ha intensificado enormemente en los últimos años (London Hazard Centre, 2010) y las mujeres lo sufren más según indica el Foro Económico Mundial (Liswood, Laura, 2015). En la cultura académica reciente se ha producido un cambio, que consiste, sobre todo, en un aumento generalizado de la productividad del profesorado. $\mathrm{Pu}-$ blicamos más y en revistas de más prestigio (que han aumentado en paralelo con nuestra presión por publicar). Así mismo, damos mayor atención al alumnado. Hemos pasado de clases convencionales y examen final (como mucho con algún parcial) a clases con interacción y con prácticas de aula y de laboratorio en carreras en las que antes ni existían. Prácticas que hemos de preparar, corregir, anotar y ponderar con el examen. A ello hemos de añadir, no sólo las tutorías, sino la docencia investigadora en la dirección de Trabajos Fin de Grado y Trabajos Fin de Máster, su corrección personalizada y la participación en tribunales para evaluarlos. Añadimos, además, la duplicación del trabajo que suponen las asignaturas semestrales (la mayoría lo son). A todo lo anterior se une el uso de las tecnologías digitales que intensifica el rendimiento del trabajo donde quiera que se instale y las universidades no son una excepción. Todas nuestras universidades disponen de campus virtuales en los cuales profesorado y alumnado vierten la mayoría de la información relevante. Tenemos, a través de estos campus, canales abiertos con el alumnado 24 horas al día, 365 días al año. No tenemos obligación de contestar a altas horas de la madrugada o en mitad de agosto, pero los correos del alumnado nos llegan y quedan pendientes y pesando en nuestro buzón de no contestados.

Esta intensificación del trabajo docente e investigador ha coincidido con la mayor entrada de las mujeres en los cuerpos docentes e investigadores de las universidades. Y si la intensificación del trabajo y la competitividad que conlleva afectan a cualquiera que la sufra, afectan particularmente a las mujeres por los roles sociales que tradicionalmente han desarrollado y porque todavía vivimos en sociedades en las que los hombres presentan notables déficits de corresponsabilidad. La terrible pandemia de Covid-19 que estamos padeciendo, mientras escribo este artículo, ha revelado de forma muy clara el diferente peso de los cuidados (domésticos, familiares, etc.) de las académicas y los académicos (Myers K.R. et al, 2020; Matthews, D., 2020). 
La mayoría de las universidades tienen muy pocas medidas o ninguna para favorecer la conciliación entre la vida profesional-académica y la vida personal y familiar. La cultura prevalente entre las personas responsables y quienes crean opinión en el mundo académico es que el PDI somos profesionales aventajados. Nuestros horarios no son de ocho horas seguidas, como la mayoría de las personas trabajadoras y apenas rendimos cuentas de lo que hacemos en nuestro horario laboral, excepto la asistencia a clases y a las tutorías. Por si esta percepción no fuera engañosa en sí misma, las universidades, por su pasado, están hechas a la medida de los hombres, son androcéntricas. Los primeros académicos, hombres ellos, tenían resuelta la logística doméstico-familiar. No necesitaban una estructura de horario y calendario ajustada a la vida fuera del campus. Con el tiempo (y las luchas por la igualdad empujando), cerca del $50 \%$ del personal docente e investigador en nuestras universidades es femenino pero la idea de que el personal académico tiene que compatibilizar la academia con otros aspectos de la vida no ha calado en nuestras centenarias instituciones. Por todo ello, las universidades no suelen tener una normativa propia de conciliación por cuidados y para compatibilizar el resto de la vida con el ejercicio de la profesión. $\mathrm{Y}$, menos aún, disponen las universidades de medidas incentivadoras de la corresponsabilidad (Liisa Husu, 2001). Las defensorías podrían proponer medidas sencillas, como prioridad para elegir horario, disminución de la carga docente, limitación horaria de reuniones de toma de decisiones, etc., En general podrían animar a revisar los sistemas de horarios, permisos, licencias y vacaciones de manera que ayudaran a mantener el rendimiento de las personas que cuiden a dependientes y su carrera profesional.

En esta línea, sugiero que se vea el baremo de gran discapacidad y se coteje con las limitaciones que supone atender a una persona dependiente (criatura o adulta) y se verá que quien cuide a una persona dependiente debiera poder acogerse a las compensaciones que tiene alguien con gran discapacidad. Porque las limitaciones para compatibilizar la vida y la profesión son aún mayores.

Y no cabe pensar sólo en el PDI y el PAS. Hay que tener en cuenta que el alumnado pueda tener personas dependientes, y también necesitarían medidas de apoyo. Algunas son muy sencillas como permitir la matrícula a tiempo parcial, la posibilidad de elegir grupo de clase, la renuncia de matrícula (total o parcial) por fuerza mayor debida a conciliación o corresponsabilidad, etc.

El impacto de las dificultades de conciliación entre profesión y familia se revela claramente en el estudio sobre brecha salarial realizado en la Universidad de Valencia (Jabbaz, Samper y Díaz, 2019). En dicho estudio se pone de manifiesto que las académicas que tienen una o más criaturas caen en un valle económico en su carrera profesional del que no se recuperaban ni cuando sus hijos se hacían mayores ${ }^{7}$ (Gráfico 4). Mientras los hombres, con hijos o sin ellos, siguen un curso ascendente sin brechas. Y no es sólo la pérdida económica, sino que esta es el reflejo de la pérdida de muchas oportunidades de desempeño profesional. No es una muestra de que las mujeres estén menos comprometidas con la universidad que sus compañeros (puede comprobarse en el similar número de sexenios por años en la institución), sino que las universidades no se comprometen con la conciliación, las parejas de las académicas tampoco y, como consecuencia, las mujeres quedan rezagadas. La penalización por la tenencia de hijos no es algo que afecte de manera especial a las investigadoras. Afecta a todas las trabajadoras, en España y fuera de ella, incluidos

7 Véase gráfico 4 
los países nórdicos, como ha puesto de relieve la reciente investigación llevada a cabo por Henrik Kleven, Camile Landais and Jakob Egholt Søgaard (2019).

Gráfico 4. Universidad de Valencia, 2015. Brecha Salarial de Género según rangos de edad y tenencia de hijas/os

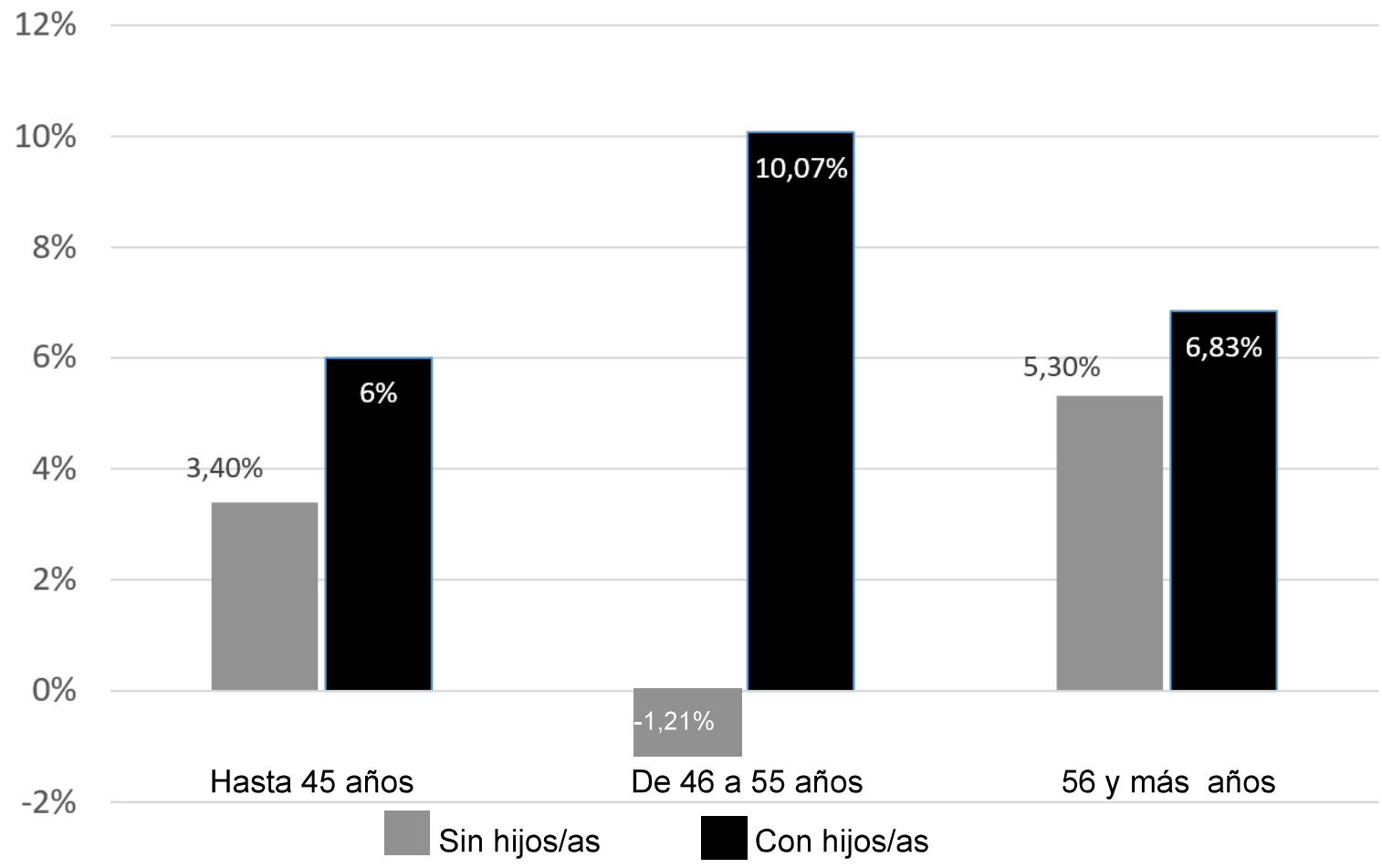

Fuente: Jabbaz, Samper y Díaz, 2019.

\section{Los contenidos androcéntricos de la ciencia y de la docencia}

La actitud ante la ciencia en nuestros días es, como poco, ambivalente. Por una parte, la ciencia es la base sobre la que pensamos el mundo (al menos en occidente) y la base sobre la que los seres humanos nos pensamos a nosotros mismos (Abraham Heschel, 1965). Por otra parte, culpamos a la ciencia por su aplicación para fines no siempre éticos (Helen Longino, 2004), por su complicidad en la destrucción medioambiental, por sus ángulos ciegos intencionados, o política de la ignorancia, (Robert Proctor y Londa Shiebinger, 2008 ,) y por su androcentrismo. Este último denunciado por numerosas científicas feministas desde hace ya unas décadas (Sandra Harding, 1996; Evelyn Fox Keller, 1991; Donna J. Haraway,1991; Londa Shiebinger, 2004; Ana Sánchez et al, 2017; Capitolina Díaz, 2018).

Las aportaciones de las científicas feministas (las arriba mencionadas y muchas otras) nos permiten afirmar que la episteme recibida y las instituciones universitarias, en sí mismas, son androcéntricas. La ciencia que hemos aprendido y el contexto en el que lo hemos hecho es el resultado de siglos de supremacía masculina, también en el campo científico. Ahora bien, este sesgo masculinista de la ciencia no es preciso que continúe. En esta segunda década del siglo XXI hemos alcanzado un grado de desarrollo, conocimiento y buenos usos democráticos que nos permiten abordar nuestras disciplinas incluyendo el 
análisis de sexo/género y transformando el conocimiento, de forma acorde con ese análisis. No hay más que ver las propuestas de la Comisión Europea para potenciar la investigación con perspectiva de género, fundamentalmente en el Programa Marco que está finalizando (Horizonte 2020 $)^{8}$ y en el próximo que ya está casi totalmente elaborado (Horizonte Europa $)^{9}$. A este respecto, las defensorías podrían hacer propuestas para estimular que en los contenidos de las asignaturas se incorpore la perspectiva de género. Pero hay que tener en cuenta que la mayoría del profesorado no ha tenido la oportunidad de recibir formación sobre docencia con perspectiva de género. Y esa es una cualificación que se tiene que trabajar para alcanzar la competencia necesaria que permita transformar la docencia (no sólo los contenidos) desde una perspectiva de género/sexo. Las publicaciones sobre docencia universitaria no sexista son escasas en cualquier lengua y, más en concreto, en español. Aunque las cosas están cambiando en este final de la segunda década del siglo XXI. Así, la Red Luis Vives de universidades de lengua catalana ha publicado una colección de guías sobre cómo incorporar la perspectiva de género en once disciplinas ${ }^{10}$, y están en proceso de difusión otras seis. Por ahora están sólo en catalán, pero parece que, en breve, alguien las traducirá al castellano y al gallego. Algunas universidades, Valencia y Extremadura, por ejemplo, han empezado a dar cursos a su profesorado sobre cómo incorporar la perspectiva de género en la docencia. Es importante que las universidades, dentro de sus objetivos de innovación y calidad, estimulen cambios en la docencia que favorezcan la igualdad de género. Es, así mismo, necesario que el propio ministerio, a través de la ANECA, garantice que todos los grados y postgrados, pero especialmente aquellos que tratan sobre seres humanos, incluyen, de forma solvente y comprobada, la perspectiva de género y que rindan cuentas sobre ello.

Los sistemas de reconocimiento oficiales de la valía del profesorado se focalizan en su valía en la investigación: su número de publicaciones, el impacto de estas, su índice $\mathrm{H}^{11}$, etc. No parecen reconocer su calidad docente. Los sistemas de reconocimiento informales (colegas y entorno académico) tampoco se centran en la docencia. Por ello, innovar en docencia, comprometerse con una docencia de calidad (que no es posible sin inclusión de la perspectiva género/sexo), no aparece como una prioridad ni para las instituciones ni para buena parte del profesorado. Por estas razones, para que el profesorado se anime a hacer el esfuerzo, primero de capacitarse y, después de trasformar su docencia con una perspectiva incluyente, necesitará estímulos que, tal vez, las defensorías puedan promover. Decíamos arriba que, hoy en día, una docencia de calidad tiene que incorporar, necesariamente, la perspectiva de género, Veamos unos pocos ejemplos que pueden ayudarnos a imaginar el valor de esta afirmación. Imaginemos cómo realizarán su trabajo las siguientes pro-

\footnotetext{
8 https://ec.europa.eu/programmes/horizon2020/en/h2020-section/promoting-gender-equality-researchand-innovation

9 https://genderaction.eu/horizon-europe/

${ }^{10}$ https://www.vives.org/book/educacio-i-pedagogia-guies-per-a-docencia-universitaria-amb-perspectivagenere/

11 "En 2005, J. E. Hirsch publicó un artículo muy citado que proponía un índice, el índice h, para estimar la importancia, el significado y el impacto, en sentido amplio, de un científico/a con respecto a sus contribuciones de investigación acumuladas. El índice h para un autor/a se basa en el mayor número de artículos incluidos que han tenido al menos el mismo número de citas que el índice. Hirsch argumentó que esto sirve para caracterizar la producción científica de un investigador y desde entonces el índice se está utilizando para comparar investigadores/as que compiten en las mismas áreas temáticas por los mismos recursos (por ejemplo, beca de investigación, posición académica o de investigación). El autor concluyó que esta era una medida imparcial de la eficiencia de publicación". (Lola Pereira y Capitolina Díaz, 2015).
} 
fesionales: a) una maestra de primaria o infantil que no haya estudiado nunca coeducación; b) una médica que no haya estudiado las diferencias de síntomas y de reacción a los medicamentos de mujeres y hombres (Bernardine Healy, 1991; Regitz-Zagrosek, Vera et al. (2016) c) una abogada que no haya tenido ni una sola asignatura en toda su carrera sobre la igualdad de género (a pesar de tener leyes sobre igualdad, desde hace décadas). Los programas de estudio de esas tres profesiones cumplen las especificidades impuestas por el ministerio correspondiente, que no obliga a incluir la perspectiva de género/sexo en el contenido de grados y postgrados. Pero tenemos leyes orgánicas que sí lo hacen. Es por tanto este, un ámbito en el que las defensorías podrían animar a la mejora de la calidad de la docencia, incluyendo en ella la perspectiva de género/sexo, y a ofertar la imprescindible formación del profesorado para llevarla a cabo.

\section{La brecha salarial de género.}

Hay una tendencia generalizada a creer que como los salarios del funcionariado se aprueban en los Presupuestos del Estado y tanto salario como complementos son iguales en cada nivel y categoría, todas y todo el funcionariado cobra lo mismo a igual nivel, categoría, antigüedad. Pero, no este no es el caso.

Hasta donde yo conozco, hay dos estudios sobre la brecha salarial de género en las universidades españolas, uno pionero, en la Universidad del País Vasco (Yolanda Jubeto y Mertxe Larrañaga, 2017) y, el segundo, el arriba mencionado, en la Universidad de Valencia. Ambos coinciden en que hay una serie de complementos que los hombres cobran y las mujeres no. En adelante, me voy a referir a este segundo. Igualando al PDI por nivel,

Gráfico 5. Universidad de Valencia, 2015. Brecha salarial ajustada por hora y categoría.

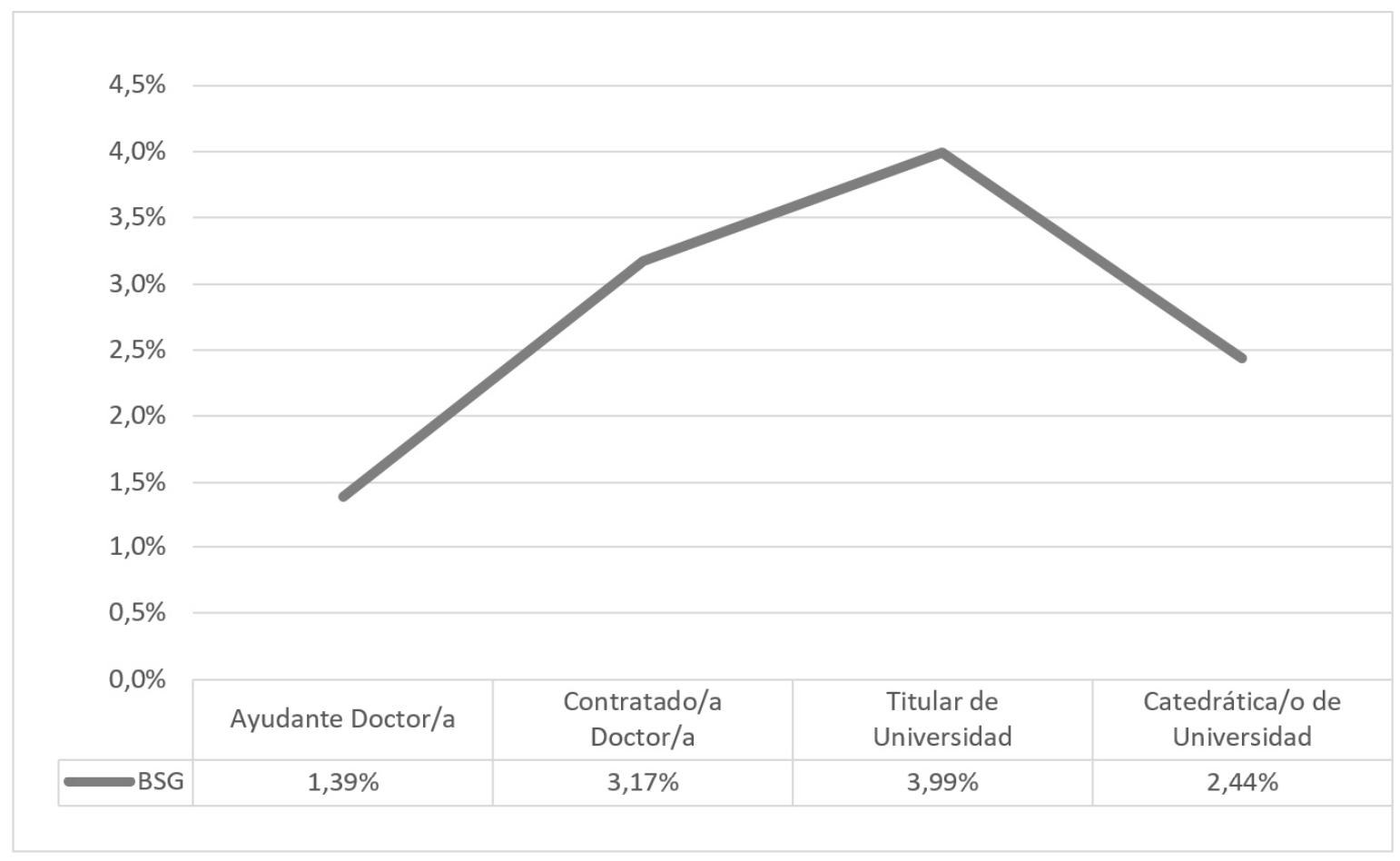

Fuente: Jabbaz, Samper y Díaz, 2019. 
categoría y horas trabajadas (brecha salarial doblemente ajustada), en la Universidad de Valencia la brecha es de 1,3\% entre ayudantes doctores, de 3,99\% entre titulares de universidad y de $2,44 \%$ en el caso de las cátedras. Si no ajustamos por categoría y horas trabajadas (brecha bruta) la brecha alcanza el 10,9\% para toda la universidad, incluido el PAS, que tiene una brecha bruta menor que el PDI. A diferencia de la mayoría de las mujeres con estudios universitarios en el mercado laboral no funcionarial, las profesoras, no cambian a tiempo parcial después de la maternidad, lo que hace que la brecha salarial en las universidades sea menor que en el empleo no funcionarial que, según el reciente estudio del Banco de España, The Child Penalty in Spain 2020 se eleva al $28 \%$.

En el gráfico 6 es interesante observar las dos columnas que muestran los complementos causantes de la mayor parte de la brecha salarial entre profesores y profesoras. La más importante está en la participación en proyectos de investigación en los cuales los o las investigadores cobran emolumentos. Son, por lo general, proyectos, contratos y convenios con terceras partes. Esos proyectos en los que el PDI cobra dinero son aquellos a los que, por lo que vemos, los colegas no llaman a las investigadoras o las entidades no firman con las mujeres tan fácilmente como lo hacen con los hombres. Algo de esto se traduce en los resultados de la primera convocatoria de sexenios por transferencia que han favorecido notablemente a los hombres (Pereira, Lola y Ana Xesús López, 2020). Pues al ser estas investigaciones para o con terceros son, por defecto, objeto de transferencia científica o tecnológica. Y los hombres que las han hecho, han tenido más oportunidades

Gráfico 6. Universidad de Valencia 2015. Diferencia salarial (€/hora) del profesorado (Titulares de Universidad). Promedio percibido por hombres y mujeres, según componentes variables salariales y por áreas de conocimiento

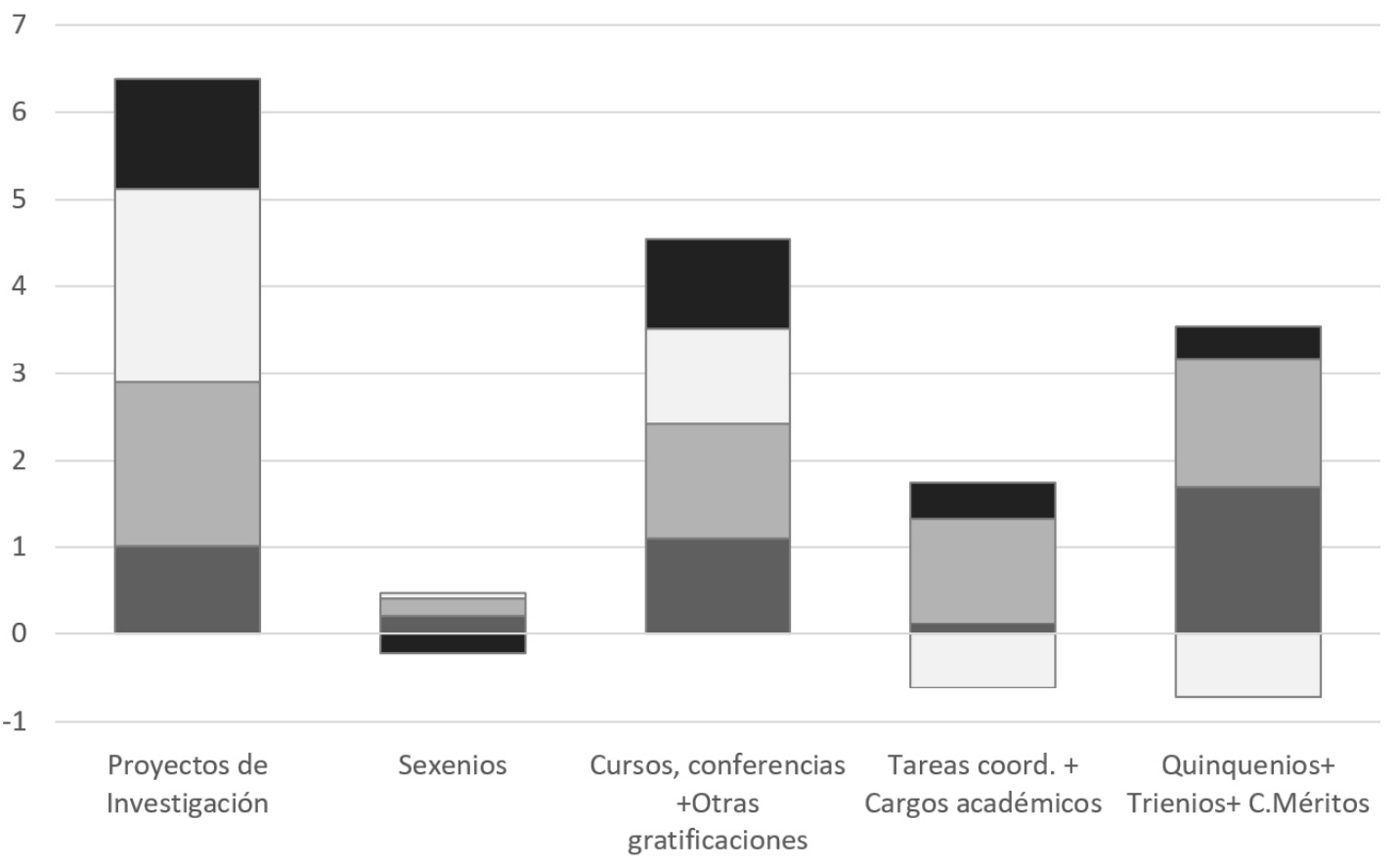

Humanidades $\square$ CCSOCIALES $\square$ CCe Ing. Bio-Medicina

Fuente: Jabbaz, Samper y Díaz, 2019. 
de alcanzar el sexenio de transferencia. Conviene no confundir esta escasa participación de las investigadoras en los proyectos en los que pueden cobrar dinero para sí mismas con su participación en proyectos de investigación pura y competitiva. En este último tipo la participación de las mujeres es muy similar a la de los hombres, como se puede ver en la equiparación en ingresos por sexenios. Las profesoras investigan tanto como los profesores, pero ya no les queda tiempo (o no las llaman) para investigar con intereses lucrativos.

La segunda columna que mejor explica la brecha salarial es la que corresponde a cursos, conferencias y otras gratificaciones. Si bien las mujeres participan bastante en tareas de coordinación y cargos académicos (participan más en los escasamente gratificados o sin pago alguno que en los más pagados), no son llamadas por su propia universidad (o no aceptan por falta de tiempo) para dar conferencias, cursos y otras actividades que suponen una gratificación suplementaria.

Identificados aquellos complementos que marcan las diferencias salariales por razones de género, las defensorías podrían animar a los órganos correspondientes de sus universidades a diseñar políticas que mitiguen esas diferencias salariales. Por lo general, tienen que ver con la falta de atención que las universidades prestan a la conciliación lo cual limita la disponibilidad horaria de las mujeres y con la "old boys network" o red académica masculina que no cuenta con las mujeres para los trabajos mejor remunerados.

\section{La escasa cultura de igualdad}

Puede que nos cueste creerlo, pero las universidades no son espacios que apuesten claramente por la igualdad, aunque puede estar en sus estatutos y tengan unidades de igualdad o instituciones similares. Las mujeres siguen padeciendo la discriminación propia de la falta de igualdad y apenas se ha mejorado en las últimas décadas desde el estudio de María Antonia García de León en 1990, en el que calificaba a las académicas como una élite discriminada. Hoy en día la discriminación está limitada por leyes que imponen medidas como la paridad en los órganos colegiados (LOMLOU, 2007), pero en la forma de cumplir esa paridad, la discriminación sigue siendo frecuente. Así, por ejemplo, muchas académicas se han visto invitadas a participar en tribunales (de tesis, de promoción, de contratación, etc.) en los que se les dice explícitamente que se les llama para cumplir la cuota femenina impuesta por la ley. Ni siquiera se adorna la petición con argumentos como la idoneidad de su perfil para lo que se la requiere. Y esto les pasa a las ayudantes doctoras y a las catedráticas. Todas son vistas y convocadas por ser mujeres. Nada más. Pocas cosas, en nuestra jerárquica academia, son tan transversales respecto a las categorías como este trato de mujeres-florero-paracumplir-la-ley al que la mayoría de nosotras nos hemos visto sometidas.

Me gustaría seguir con los ejemplos, pues creo que este epígrafe queda mejor ilustrado con ejemplos específicos. Después de las mujeres-florero-para-cumplir-la-ley, veamos el caso de la segregación horizontal en los grados universitarios. Es un ejemplo que sirve de muestra de cómo las universidades ignoran la desigualdad de género que ellas mismas reproducen e incluso, en algunos casos, amplían. Las universidades llevan mucho tiempo viendo como los chicos huyen de carreras como magisterio, trabajo social o enfermería - entre otras- y cómo las chicas apenas se acercan a las ingenierías, a física o a filosofía (Gráfico 7). 
Gráfico 7. Evolución del porcentaje de alumnas matriculadas en Grado, 1er Ciclo de universidades públicas según ramas de enseñanza. Cursos 2012-13 a 2016 -17

Porcentaje de muejres sobre el total de cada rama de la enseñanza

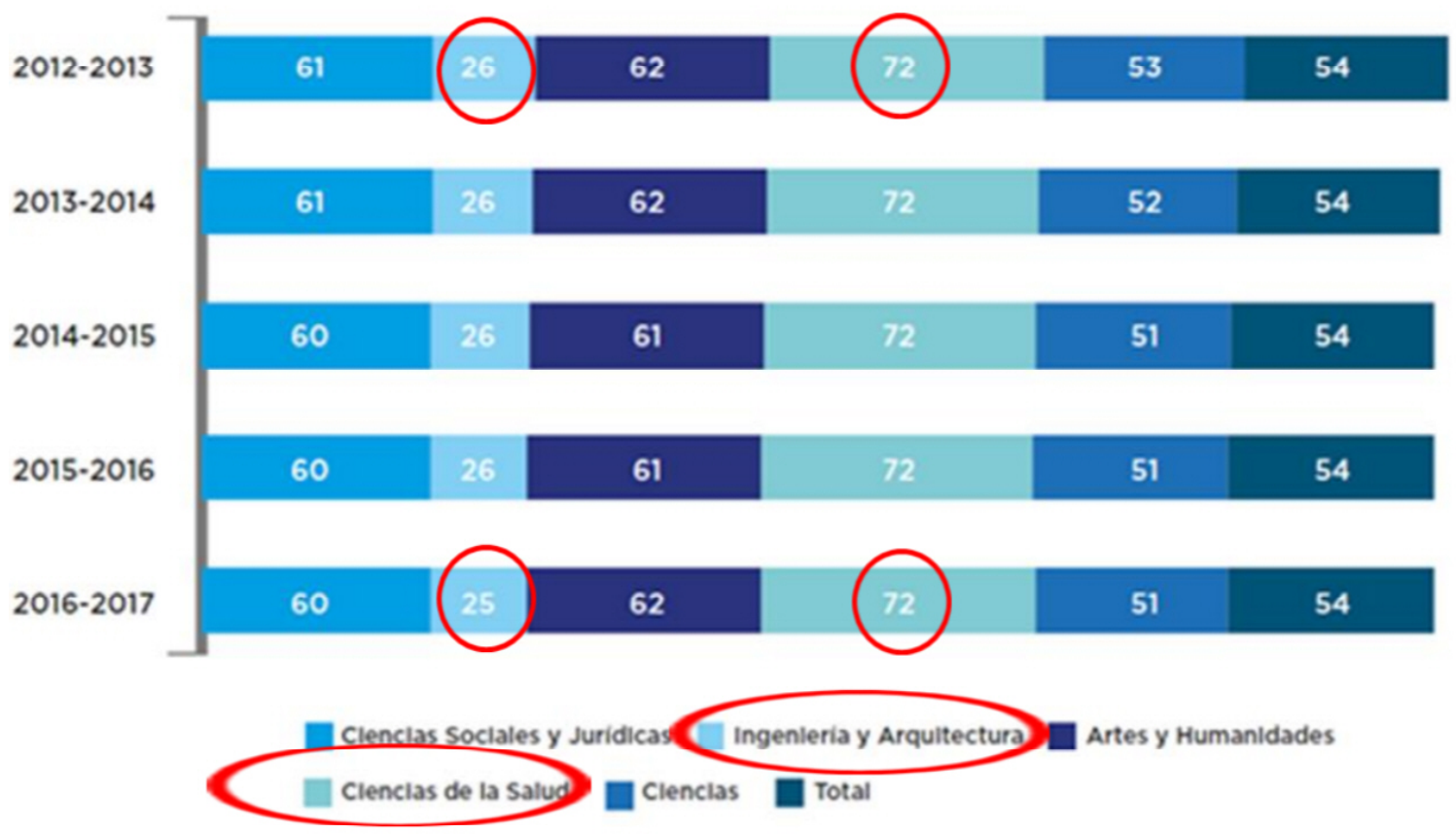

Fuente: Científicas en cifras, 2016.

¿Alguien se ha planteado si esto es un problema? ¿No sería mejor hacer algo para que las chicas también participaran en las carreras técnicas y los chicos en las de cuidados? ¿No es mejor que haya más diversidad en todas partes? (Hallberg, Nicole and Martin Schneider, 2017). Los conocimientos y el ejercicio de las profesiones probablemente se ven afectados por ser practicados por un solo tipo de personas, un solo género. Es imposible no introducir los sesgos propios del género dominante en un campo determinado. Se produce una suerte de identificación entre profesión y profesional. Cuando la profesionalidad sólo se encarna en un sexo/género, esta se va definiendo con características propias de la cultura y la autopercepción propia de ese género que la ocupa casi en exclusiva. Si la diversidad es una ventaja en los ecosistemas naturales, aún lo es más en los sociales. La universidad, como productora y reproductora de conocimiento y buscadora de la excelencia, debiera notar las ventajas de una cultura paritaria.

Otro ejemplo, ¿se sorprende alguien y trata de evitar que, en una inauguración de curso, en la presidencia de un congreso científico, de una exposición, etc. sólo haya hombres en la mesa presidencial? ¿se preocupa alguien de que en un congreso realizado y subvencionado por la universidad todos los miembros del comité científico sean hombres? ¿Alguien ha pensado si las minorías con identidades de género más allá de mujeres y hombres cisgénero están representadas en algún espacio? Naturalmente estás son preguntas retóricas que espero sirvan para que las defensorías se las hagan (estas y muchas otras similares) para saber cómo son sus universidades en asuntos de des/igualdad de género y puedan servirles como pistas para saber por dónde enfocar algunas soluciones. 
Convendría, por todo lo antedicho, que las defensorías tengan un papel proactivo $\mathrm{y}$ vigilante para ir corrigiendo las discriminaciones de género que continuamente se producen y para prevenir aquellas que sabemos que es fácil que aparezcan.

\section{Referencias}

Barker, Gary, Christine Ricardo y Marcos Nascimento (2007). Engaging men and boys in changing gender-based inequity in health: Evidence from programme interventions. Ginebra: Organización Mundial de la Salud (WHO). https://www.who.int/gender/documents/Engaging_men_boys.pdf

Benson, Donna J. y Gregg E. Thomson (1982). "Sexual Harassment on a University Campus: The Confluence of Authority Relations, Sexual Interest and Gender Stratification". Social Problems, Volume 29, Issue 3, Pages 236-251.

Bondestam Fredrik and Maja Lundqvist (2020a). "Sexual harassment in higher education. A systematic review", European Journal of Higher Education. http://www.tandfonline. com/doi/pdf/10.1080/21568235.2020.1729833

Bondestam, Fredrik and Maja Lundqvist (2020b). Efforts to Prevent Sexual Harassment in Academia. An International Research Review. Stockholm: The Swedish Council for Higher Education.

Bonhet, Iris (2018). Lo que sí funciona. Cambios de conducta para proyectar la igualdad de género. México:Grano de Sal; Edición.

Díaz Capitolina y Sandra Dema (2013). "Las mujeres y la ciencia. La escasez de mujeres en la academia. Un caso de histéresis social".10ocias@uned, no6, pp.149-156.

Dessel, A. B., K. D. Goodman, and M. R. Woodford (2017). "LGBT Discrimination on Campus and Heterosexual Bystanders: Understanding Intentions to Intervene." Journal of Diversity in Higher Education, 10: 101-116.

Díaz Capitolina (coord.) (2016). SheScience: ciencia con perspectiva de género. Mètode: Revista de difusión de la Investigación, $n^{\circ} 91$.

Díaz Martínez Capitolina (2018). "Mujeres de ciencias, mujeres en las ciencias, ciencia con mujeres". En Rita Radl Philipp y Ana Elizabeth Santos Alves (eds.). Memoria, género y educación: investigaciones y cuestiones epistemológicas. Santiago de Compostela: Universidade de Santiago de Compostela, págs. 93- 106.

Durán María Ángeles (1981). "Una ausencia de mil años: la mujer en la universidad". En Durán, $M^{\mathrm{a}}$ Ángeles (coord.) La mujer en el mundo contemporáneo. Madrid: Universidad Autónoma de Madrid, págs.: 53-67.

EU. Standing Working Group on Gender in Research and Innovation (2020). Sexual Harassment in the Research and Higher Education Sector National Policies and Measures in EU Member States and Associated Countries. https://genderaction.eu/wp-content/uploads/2020/06/SWGGRI_Sexual-Harassment-in-the-Research-Higher-Ed.-National-Policies-Measures.pdf

Fox Keller, Evelyn (1991). Reflexiones sobre género y ciencia. Valencia: Edicions Alfons el Magnanim.

García de León, María Antonia (1990). "Las profesoras universitarias: el caso de una élite discriminada". Revista Complutense de Educación 10 (2): 355-372.

Geldschläger, Heinrich et al. (2010). "Programas Europeos de Intervención para Hombres que Ejercen Violencia de Género: Panorámica y Criterios de Calidad", Psychosocial Intervention, vol.19 no.2. 
Hallberg, Nicole and Martin Schneider (2017), "Sexism in the workplace is real: a story from two perspectives", https://www.vox.com/first-person/2017/3/17/14950296/ sexism-name-switch-tweets

Haraway, Donna J. (1991). Ciencia, cyborgs y mujeres. La reinvención de la naturaleza, Madrid: Cátedra.

Harding, Sandra (1996). Ciencia y feminismo. Madrid: Morata.

Heschel, Abraham (1965). Who is man? Stanford: Stanford University Press.

Hirsch J. E. (2005). "An index to quantify an individual's scientific research output" Proceedings of National Academy of Sciences U S A (PNAS). Nov 15; 102(46): 16569-16572.

Husu, Liisa (2001). Sexism, Support and Survival in Academia. Academic Women and Hidden Discrimination in Finland. Helsinki: University of Helsinki.

Jabbaz, Marcela, Samper Teresa y Díaz Capitolina (2019), "La brecha salarial de género en las instituciones científicas. Un estudio de caso", Convergencias. Revista de Ciencias Sociales, 80: 1-27.

Johnson, P., S. Widnall, and B. Frazier (2018). Sexual Harassment of Women. Climate, Culture, and Consequences in Academic Sciences, Engineering, and Medicine. Washington: National Academy of Sciences.

Jubeto, Yolanda y Mertxe Larrañaga (2017). Presupuestos con enfoque de género en la UPV/EHU (2011-2016). https://www.ehu.eus/es/web/berdintasuna-direccionparalaigualdad/aurrekontuak-genero-ikuspegiarekin?inheritRedirect=true y https://www.ehu.eus/es/web/berdintasuna-direccionparalaigualdad/aurrekontuak-genero-ikuspegiarekin?inheritRedirect=true

KLeven, Henrik, Camile Landais and Jakob E. Søgaard (2019). "Children and Gender Inequality: Evidence from Denmark", American Economic Journal: Applied Economics, 11(4), 181-209.

Liswood, Laura (2015), "Work stress: why women have it worse than men", World Economic Forum, https://www.weforum.org/agenda/2015/10/work-stress-womenhave-it-worse/

London Hazard Centre (2010). FAS Stress at Work. Londres: London Hazard Centre.

MacKinnon, Catharine, A. (1979). Sexual Harassment of Working Women: A Case of Sex Discrimination. New Haven, CT: Yale University Press.

Marks, M. J., \& Fraley, R. C. (2006), "Confirmation bias and the sexual double standard." Sex Roles 54(1/2), 19-26.

Matthews David (2020). "Pandemic lockdown holding back female academics, data show", Times Higher Education, June 20. https://www.timeshighereducation.com/news/pandemic-lockdown-holding-back-female-academics-data-show

Mederos, F. (2002). "Changing our visions of intervention, the evolution of programs for physically abusive men". En E. Aldarondo, \& F. Mederos (Eds.), Men who batter: Intervention and prevention strategies in a diverse society. New York: Civic Research Institute.

Myers, Kyle R. et al. (2020). "Unequal effects of the covid-19 pandemic in scientists", Nature Human Behaviour , 1-4. https://www.nature.com/search?q=Unequal+effects + of +the+covid-19+pandemic+in+scientists

Nuño Gómez, Laura y Enrique Álvarez Conde (2017). "Androcentrismo académico: la ficción de un conocimiento neutral". En Marcos Jesús Iglesias Martínez e Inés Lozano Cabezas (coords.), La (in)visibilidad de las mujeres en la Educación Superior: retos y desafíos en la Academia. Feminismo/s, 29: 279-297.

Organización Mundial de la Salud (WHO) (2009). Violence Prevention. The evidence. Promoting gender equality to prevent violence against Women. 
https://www.who.int/violence_injury_prevention/violence/gender.pdf

Nickerson Raymond S. (2006), "Confirmation bias: A ubiquitous phenomenon in many guises." Review of General Psychology 2(2), 175-220.

Parlamento Europeo (2014). Proyecto de Informe sobre carreras profesionales en los ámbitos científico y académico y los techos de cristal existentes (2014/2251 (INI)). Comisión de los Derechos de la Mujer e Igualdad de Género. Ponente: Elissavet Vozemberg. Bruselas-Estrasburgo: Parlamento Europeo.

Pereira, Lola y Díaz Capitolina (2016). "Are Women Properly Represented in Scientific Publications and Research? Interim results from a Spanish Case Study in Earth Sciences", Episodes. Journal of International Geosciences, Vol 38-9 (1): 52-58.

Pereira, Lola y Ana Xesús López (2020). "Transferencia del conocimiento: ¿Estamos haciendo bien las cosas las investigadoras españolas?", El País, 10 de julio. https:// elpais.com/ciencia/2020-07-10/transferencia-del-conocimiento-estamoshaciendo-bien-las-cosas-las-investigadoras

Phyllis B. Frank, M.A. and Gail K. Golden, M.S.W., Ed.D. (2002). Mental Health Treatment with Men Who Batter. http://www.goldenwrites.com/MentalHealthTreatmentMB.pdf. Consultado el 12/07/2020

Proctor, Robert y Londa Schiebinger (Eds.) (2008). Agnotology: The Making and Unmaking of Ignorance. Stanford: Stanford University Press.

Regitz-Zagrosek, Vera et al. (2016). Gender in cardiovascular diseases: impact on clinical manifestations, management, and outcomes, European Heart Journal, Volume 37, Issue 1, 1 January 2016, Pages 24-34, https://doi.org/10.1093/eurheartj/ehv598

Reilly, M.E., Lott, B. \& Gallogly, S. M. (1986). "Sexual harassment of university students". Sex Roles 15, 333-358 (1986).

Sánchez, Ana et al. (2017). "An open path for gender equality in research: when female scientists question the state of science and the institutions embrace the criticism". Mètode Science Studies Journal: Annual Review, No 7, págs.96-103.

Selma Penalva, Alejandra (2019). "La conciliación en la universidad, ¿un asunto solo de mujeres?", Revista de Educación y Derecho, $\mathrm{n}^{\circ}$ 20, https://revistes.ub.edu/index.php/ RED/article/view/29703/30031

Shiebinger, Londa (2004) ¿Tienes sexo la mente? Madrid: Cátedra.

Smith Brendan L. (2018). "What it really takes to stop sexual harassment. Psychologists call for a comprehensive approach with real-world impact". Good Company, APA's newsletter, Vol 49, No. 2.

SWG GRI. 2020. Sexual Harassment and Higher Education: National Policies and Measures in EU Member States. ERAC Standing Working Group on Gender in Research and Innovation.

The World Bank's (2015), Mind, Society and Behaviour. http://www.worldbank.org/en/ publication/wdr2015

Unidad de Igualdad UCM (2018). Estudio sobre acoso sexual, acoso sexista, acoso por orientación sexual y acoso por identidad y expresión de género en la Universidad Complutense de Madrid, 2018. https://www.ucm.es/data/cont/media/www/pag-3331/Resumen\%20 resultados\%20Estudio_Acoso.pdf 